\title{
Enroute Airspace Control and Controller Workload Analysis using a Novel Slot-based Sector Model ${ }^{1}$
}

\author{
Kaushik Roy ${ }^{2}$
}

\begin{abstract}
A novel slot-based sector structure is introduced and applied to enroute airspace control. Aircraft entering a sector are assigned trajectories to safely align to an assigned slot. Aircraft move through the rest of the sector, maintaining their slot at nominal velocity. Safety is provided according to proper spacing and assignment of slots. Analysis of singlelink sectors with periodic arrival flows shows the proposed alignment control strategies safely handle several aircraft at a given altitude; the vector-for-spacing strategy outperforms strategies utilizing only velocity changes. Wide variations in worst-case capacities are observed under more general arrival processes; capacities are observed to increase with reduced uncertainty and with increased outflow spacing. Finally, a controller workload metric is developed and its use illustrated; we see that complex alignment strategies and uncertainty in arrivals increases workload.
\end{abstract}

\section{INTRODUCTION}

As air travel reaches new highs in the United States, optimization of air traffic becomes more necessary. The highly interconnected nature of the National Airspace System (NAS), coupled with the possibility of poor weather, leads to highly variable flow in the busiest air traffic corridors, such as the Northeastern United States. Variability, along with increasing use of limited airspace, makes tactical control of enroute traffic complex and stressful to air traffic controllers.

Current work in enroute control has focused on three main topics: strategic flow control, tactical conflict detection and resolution, and 4-dimensional trajectory contracts. Strategic flow control has been posed as an optimization problem [1], [2], where the goal is to maximize total flow or minimize delay while meeting safety constraints. Solutions tend not to deal with local control policies used to separate aircraft. Tactical conflict detection and resolution focuses on the other end of the spectrum, incorporating optimal control ideas, geometric arguments, and a variety of other methods to ensure safe separation between aircraft locally [3], [4], [5], [6]. A newer idea is that of $4 \mathrm{D}$ contracts, in which safe trajectories are assigned to a set of aircraft [7], [8], [9]. Ideally, 4D contracts would replace the separate processes of flow control and conflict detection and resolution. In the above methods, a key idea is reduction of controller workload through automated support tools. Indeed, controller workload is extremely relevant to air traffic optimization; an understanding of controller workload is necessary for development

\footnotetext{
${ }^{1}$ This research was supported by ONR under the CoMotion MURI contract N00014-02-1-0720, by NASA JUP under grant NAG2-1564, and by NASA grant NCC2-5536

${ }^{2}$ Ph.D. Candidate, Electrical Engineering, Stanford University.

${ }^{3}$ Associate Professor, Aeronautics and Astronautics, Courtesy Associate Professor, Electrical Engineering; Director, Hybrid Systems Laboratory, Stanford University.
}

of practical algorithms and tools. Previous work has focused on workload metrics based on theoretical arguments or to match controller surveys [10], [11], [12], [13], [14].

In this paper, we focus on developing structure in enroute air traffic control to maintain efficient, safe flows without overburdening controllers. A novel slot-based structure is proposed for the control of individual enroute airspace sectors in Section II. The structure consists of generating safe trajectories for aircraft as they enter sectors to ensure separation is maintained with minimal effort throughout the remainder of the sector. Sections III and IV discuss the capacity limits obtained under fixed-spacing and general arrival processes, respectively. Controller workload results are discussed in Section V. Finally, conclusions and future work directions are presented in Section VI.

\section{A SLOT-BASED SECTOR MODEL}

The NAS is organized hierarchically, with 22 centers each subdivided into about 20 sectors. Sectors cover regions of airspace on the order of several hundred square miles. Enroute sectors are also stratified vertically. Aircraft paths are generally along predefined jetways; tactical control is used to maintain safe separation. The number of aircraft allowed in a sector is therefore limited by the ability of human controllers to detect and resolve conflicts. Safe separation in enroute airspace is defined as no aircraft within $5 \mathrm{~nm}$ laterally or $1000 \mathrm{ft}$ vertically of any other aircraft.

Several techniques have become the norm for maintaining enroute air traffic. For example, control is often implemented as aircraft enter the sector so that these aircraft can subsequently be monitored less frequently. Velocity changes are used to make small changes in aircraft spacing. Flight path length changes, either longer (vector-for-spacing) or shorter (direct-to), are used to alter aircraft spacing and to facilitate the merging of multiple air traffic flows. Altitude changes are used, though less frequently, in enroute airspace. A single direction of flow at a specific altitude is assumed in the paper. In real traffic, flows in opposite directions occupy alternating flight levels. Holding patterns are not generally used in enroute airspace, but the tool remains available if safety would otherwise be compromised.

In designing an improved sector structure, these controller practices are mirrored. Figure 1 presents a schematic of the proposed sector structure. The central idea is that of slots moving at nominal velocity in the latter portion of the sector, denoted the maintenance phase. In this region of airspace, aircraft maintain their position on a slot at nominal velocity. Slots are spaced appropriately to ensure 
safe separation between aircraft, even in complex scenarios such as merging traffic. Aircraft are assigned a unique slot upon entry to the sector in a first-come first-served manner. Predetermined control policies are designed to ensure safe separation between aircraft in the alignment phase and to ensure aircraft are coincident in position and velocity with their assigned slot as they enter the maintenance phase.

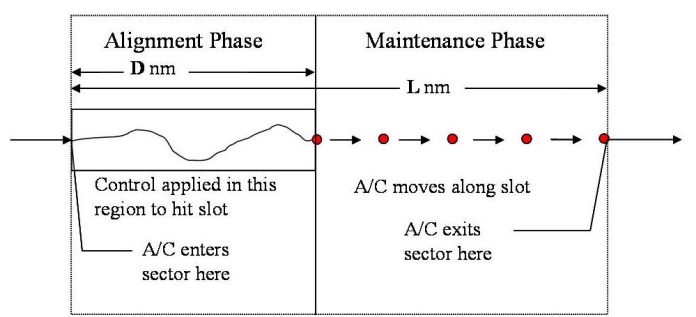

Fig. 1. Schematic of slot-based model for a single-link sector. The model can be extended to multiple entries and exits by applying the alignment phase to each entry link.

Inefficiencies in the structure can be reduced through several auxiliary methods. For example, slot positions can be reset after a gap in traffic, and direct-to routing can be used to shorten the path of otherwise unimpeded traffic.

The following subsections describe three possible control policies for alignment. The proposed policies generate piecewise linear, piecewise constant velocity trajectories. More realistic trajectories would be used to implement this scheme in real scenarios. The designed trajectories through the alignment phase are 1) fixed velocity, 2) nominal followed by slow phase, and 3 ) vector-for-spacing. In all cases, it is assumed aircraft travel at nominal velocity just before entering the sector and throughout the maintenance phase.

\section{A. Fixed velocity}

Aircraft are assumed to maintain velocities within $10 \%$ of nominal (i.e. - $[540,660] \mathrm{nm} / \mathrm{hr}$ ). The control policy simply consists of determining the constant velocity necessary to reach the end of the alignment phase at the same time as its assigned slot. Trajectories are along the link and determined completely by entry time and assigned velocity.

\section{B. Piecewise combination of nominal and low velocity}

This control policy consists of a flight segment at nominal speed followed by a segment at the lowest allowed speed (540 nm/hr). Given an entry time and a desired exit time from the alignment phase, the length of each segment can then be determined. Trajectories are along the link and set by entry time and the nominal velocity segment length.

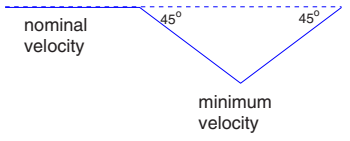

Fig. 2. Schematic of vector-for-spacing trajectory.

\section{Vector-for-spacing}

The third alignment phase control policy involves lengthening the flight path. Aircraft in the alignment phase fly a nominal velocity segment followed by a vector-for-spacing segment at minimum velocity, $540 \mathrm{~nm} / \mathrm{hr}$. The spacing maneuver involves a $45^{\circ}$ turn away from the link, then a $90^{\circ}$ turn toward the link, as sketched in Figure 2. Trajectories are assumed to be piecewise linear. The spacing segment angles can be variable but are set for this paper.

\section{APPLICATION OF SLOT-BASED MODEL TO FIXED-SPACING ARRIVAL PROCESS}

In this section, the structure proposed in Section II is applied to scenarios where aircraft enter with fixed spacing. This simplification aids in understanding the slot-based model and also provides a segue to the next section, where more realistic flows are treated.

Enroute controllers are often required to ensure a "smooth" flow to downstream sectors, which may involve reducing aircraft-spacing variation or adding delay to meet miles-intrail or minutes-in-trail restrictions. The capabilities of the slot-based model are better understood through its limits; that is, the worst-case scenario that can be handled under certain assumptions of the sector and the incoming traffic. For a given arrival process, worst-case is defined as the maximal number of aircraft which can be safely controlled before available control policies are no longer feasible. More drastic control options such as altitude changes or holding patterns would be required for further aircraft. Results are developed for a sector with a single link connecting an entry point and an exit point.

The arrival process is denoted fixed-spacing: the spacing between aircraft is constant. The aircraft are delivered to a downstream sector with another, possibly different, fixed spacing. Assuming downstream spacing is larger than arrival spacing, the situation corresponds to adding delay to reduce overall flow out of the sector. Control becomes perfunctory if downstream requirements are less restrictive than upstream requirements.

Consider a single-link sector in which aircraft enter every $\alpha$ seconds and exit every $\beta$ seconds, with $\alpha<\beta$. Worst-case analysis is to determine the number of consecutive aircraft that can be delivered to the downstream sector without violating minimum separation or velocity requirements. Each of the three control policies described in the previous section are treated below.

The slot-based sector structure applied to the single-link sector yields the schematic in Figure 1. The sector is treated as a single link of length L (in relevant units, such as nautical miles). The first D nm of the link are the alignment portion. Consider an inflow of $N$ identical aircraft, wholly described by their entry times: $t_{i}, i \in 0, \ldots, N-1$. Throughout this discussion, the variables $x_{i}(t)$ and $v_{i}(t)$ are used to refer to the position and velocity of aircraft $i$, respectively. Slots move at nominal velocity and have position $s_{j}(t)$. Times are in seconds unless otherwise specified.

The fixed-spacing arrival process, with parameter $\alpha$, means aircraft 0 enters the system at time $t_{0}=0$, aircraft 1 at time $t_{1}=\alpha$, and aircraft $i$ at time

$$
t_{i}=\alpha i
$$

for $i \in 0,1, \ldots, N-1$. Slots are defined by the first aircraft in the system. Aircraft 0 is allowed to transit the sector at nominal velocity $\left(v_{0}=v_{n o m}=600 \mathrm{~nm} / \mathrm{hr}=1 / 6 \mathrm{~nm} / \mathrm{sec}\right)$. Its position can be written as $x_{0}(t)=t / 6$. Since aircraft 
0 moves at nominal velocity, its location is labelled slot 0 (that is, the position of slot 0 can be defined as $s_{0}(t)=$ $\left.x_{0}(t)=t / 6\right)$. Other slots can then be assigned with the goal to deliver aircraft to downstream sectors with $\beta$ second spacing (at nominal velocity, $\beta / 6 \mathrm{~nm}$ spacing). Therefore, slot $j$ has position $s_{j}(t)=(t-j \beta) / 6$.

Control in the alignment phase requires matching boundary conditions. The aircraft must enter the sector at the proper time, $x_{i}\left(t_{i}\right)=0$, and must exit the alignment phase at the same time as its assigned slot, $x_{i}\left(T_{i}\right)=s_{i}\left(T_{i}\right)$, where $T_{i}$ is the time aircraft $i$ exits the alignment phase. Because the alignment phase is $D \mathrm{~nm}$ in length, $T_{i}$ is found by solving $s_{i}\left(T_{i}\right)=D$, yielding

$$
T_{i}=6 D+\beta i
$$

\section{A. Fixed velocity trajectories}

The fixed velocity control policy assigns aircraft in the alignment phase a velocity within $10 \%$ of nominal. The appropriate trajectory for aircraft $i$ is to maintain constant velocity

$$
v_{i}(t)=600 \frac{6 D}{6 D+i(\beta-\alpha)}
$$

during the alignment time interval, $t \in\left[t_{i}, T_{i}\right]$. This velocity ensures aircraft hit their assigned slot at time $T_{i}$.

The trajectory is allowable if the following requirements are met: (1) aircraft velocity constraints and (2) separation requirements between aircraft $i-1$ and $i$. Case (1) requires $v_{i} \in[540,660]$; the active constraint is $v_{i} \geq 540$. Combining this constraint with Equation (3) yields

$$
i \leq \frac{2 D}{3(\beta-\alpha)}
$$

Case (2) requires aircraft $i-1$ is at least $5 \mathrm{~nm}$ into the sector when aircraft $i$ enters; that is, $x_{i-1}\left(t_{i}\right) \geq 5 \mathrm{~nm}$, which yields

$$
i \leq D \frac{\alpha-30}{\beta-\alpha}+1
$$

Aircraft $i$ can safely transit the sector if (4) and (5) are met. This implies the maximal number of aircraft that can be handled by the slot-based sector structure is

$$
1+\left\lfloor\min \left(\frac{2 D}{3(\beta-\alpha)}, D \frac{\alpha-30}{\beta-\alpha}+1\right)\right\rfloor .
$$

These results and similar results for the other control policies are found in Table I. Table II shows reasonable combinations of $\alpha$ and $\beta$ and associated worst-case values. An example in which the control structure breaks down is shown in Figure 3. This sector has length $L=100 \mathrm{~nm}$, alignment distance $D=20 \mathrm{~nm}$, and $(\alpha, \beta)=(40,41)$ seconds. Table II states that 14 aircraft can safely transit the sector; the $15^{\text {th }}$ aircraft (aircraft 14) would travel at $537.3 \mathrm{~nm} / \mathrm{hr}$, below the minimum allowed $540 \mathrm{~nm} / \mathrm{hr}$. Therefore, other control strategies would be needed for more than 14 aircraft.

\section{B. Piecewise combination of nominal and slow trajectories}

The second proposed control policy is characterized by the location of the transition from nominal to minimum velocity, denoted $d_{i}$. The entry and exit times for the alignment phase for aircraft $i$ are derived in (1) and (2). To solve for $d_{i}$, note that traversal of the alignment phase takes $\frac{d_{i}}{600}+\frac{D-d_{i}}{540}$ hours; converting into seconds and equating with $T_{i}-t_{i}$ yields

$$
d_{i}=D-3(\beta-\alpha) i / 2 \text {. }
$$

The distance $d_{i}>0$; otherwise, aircraft $i$ must incur more delay than is possible. This translates into

$$
i \leq \frac{2 D}{3(\beta-\alpha)} \text {. }
$$

A second requirement is that aircraft $i-1$ be at least $5 \mathrm{~nm}$ into the sector when aircraft $i$ enters the sector: $x_{i-1}\left(t_{i}\right) \geq 5$ $\mathrm{nm}$, which yields

$$
i \leq \frac{2 D+3 \alpha-100}{3(\beta-\alpha)}+1
$$

The worst-case capacity is again one more than the floor of the minimum of the right sides of (8) and (9). Refer to Table I to compare these results to the other control policies.

\section{Vector-for-spacing trajectories}

This control policy is also characterized by the point where the nominal velocity segment ends, denoted $d_{i}$. Computation of $d_{i}$ follows the same analysis as for the previous control policy, except the spacing segment corresponds to an alongtrack velocity of $540 / \sqrt{2} \mathrm{~nm} / \mathrm{hr}$. The resulting constraints on the feasibility of aircraft $i$ are listed in Table I.

TABLE I

EXPRESSIONS IN TABLE REPRESENT CONDITIONS IN WHICH AIRCRAFT $k$

\begin{tabular}{|l|c|c|}
\hline $\begin{array}{l}\text { Control } \\
\text { Policy }\end{array}$ & $\begin{array}{c}\text { Physically } \\
\text { infeasible } \\
\text { trajectory }\end{array}$ & $\begin{array}{c}\text { Conflict } \\
\text { between aircraft } \\
i \text { and } i-1\end{array}$ \\
\hline 1) Fixed-velocity & $\frac{2 D}{3(\beta-\alpha)}$ & $\frac{D(\alpha-30)}{\beta-\alpha}+1$ \\
\hline 2) Nominal/slow & $\frac{2 D}{3(\beta-\alpha)}$ & $\frac{2 D+3 \beta-100}{3(\beta-\alpha)}$ \\
\hline 3) Vector-for-spacing & $\frac{(20 \sqrt{2}-18) D}{3(\beta-\alpha)}$ & $\frac{(20 \sqrt{2}-18) D+3 \beta-100 \sqrt{2}}{3(\beta-\alpha)}$ \\
\hline
\end{tabular}

TABLE II

WORST-CASE CAPACITIES FOR SLOT-BASED SECTOR MODEL FOR A SINGLE-LINK SECTOR UNDER A VARIETY OF PARAMETERS $D, \alpha, \beta$ AND

\begin{tabular}{|c|c||cc|c|c||c|c|c|}
\hline \multicolumn{3}{|c|}{$D$} & Control & $\alpha:$ & \multicolumn{3}{|c|}{40} & \multicolumn{3}{|c|}{60} \\
\cline { 2 - 9 } & Policy & $\beta:$ & 41 & 45 & 60 & 61 & 65 & 80 \\
\hline \multirow{3}{*}{20} & 1 & & $\mathbf{1 4}$ & $\mathbf{3}$ & $\mathbf{1}$ & $\mathbf{1 4}$ & $\mathbf{3}$ & $\mathbf{1}$ \\
\cline { 2 - 9 } & 2 & & $\mathbf{1 4}$ & $\mathbf{3}$ & $\mathbf{1}$ & $\mathbf{1 4}$ & $\mathbf{3}$ & $\mathbf{1}$ \\
\cline { 2 - 9 } & 3 & & $\mathbf{6 3}$ & $\mathbf{1 4}$ & $\mathbf{4}$ & $\mathbf{6 9}$ & $\mathbf{1 4}$ & $\mathbf{4}$ \\
\hline \multirow{3}{*}{100} & 1 & & $\mathbf{6 7}$ & $\mathbf{1 4}$ & $\mathbf{4}$ & $\mathbf{6 7}$ & $\mathbf{1 4}$ & $\mathbf{4}$ \\
\cline { 2 - 9 } & 2 & & $\mathbf{6 7}$ & $\mathbf{1 4}$ & $\mathbf{4}$ & $\mathbf{6 7}$ & $\mathbf{1 4}$ & $\mathbf{4}$ \\
\cline { 2 - 9 } & 3 & & $\mathbf{3 3 7}$ & $\mathbf{6 9}$ & $\mathbf{1 8}$ & $\mathbf{3 4 3}$ & $\mathbf{6 9}$ & $\mathbf{1 8}$ \\
\hline
\end{tabular}

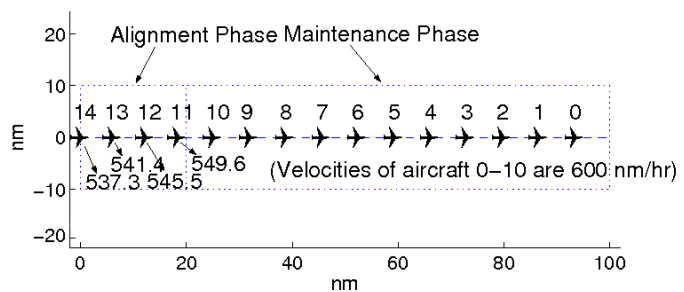

Fig. 3. Failure of slot-based sector model as predicted by expressions of worst-case scenarios. The fixed-velocity control policy is used for a singlelink sector with length $L=100 \mathrm{~nm}$, alignment phase length $D=20 \mathrm{~nm}$, and spacing parameters $(\alpha, \beta)=(40,41)$ seconds. Labels are above each aircraft, while velocities are given below.

Single-link sector results are extended to sectors with more complicated network structure, such as a sector with two entry and exit points. However, these results are omitted for space considerations. 


\section{APPLICATION OF SLOT-BASED MODEL TO GENERAL-ARRIVAL PROCESS}

In this section, the slot-based sector model is applied to sectors with more general arrival processes. Real air traffic exhibits uneven inflow, but the goal remains to deliver an even outflow to the downstream sector. The worst-case capacity is expressed as a probability distribution on the maximum number of aircraft which can be controlled using the slot-based sector model (for a given statistical description of the arrival process). Simulation results follow the derivation of worst-case expressions.

Assume $N$ aircraft with arrival times $t_{i}$ for $i \in\{0, \ldots, N-$ $1\}$, with $t_{0}=0$ and $t_{i}<t_{j}$ for $i<j$, rather than $t_{i}=\alpha i$ as in Section III.

Constraints $\mathbf{C}$ For each of $N$ aircraft entering a sector, a set of constraints must be met for the aircraft to transit the sector safely. For a given aircraft $i$, the $m$ constraints are denoted $C_{i, j}$, for $j \in\{1, \ldots, m\}$. Note that $i \in\{1, \ldots, N-1\}$, since aircraft 0 always transits safely.

At least the first $k$ aircraft can transit the sector safely if all $m(k-1)$ constraints $C_{i, j}, i \in\{1, \ldots, k-1\}, j \in\{1, \ldots, m\}$ are met. Exactly $k$ aircraft can transit the sector safely if, in addition, aircraft $k$ cannot; i.e. - if at least one of $C_{k, j}$ is false for $j \in\{1, \ldots, m\}$. Events $C_{i, j}$ and $C_{k, l}$ are in general not independent for arbitrary $i, j, k, l$. A constraint $C_{i, j}$ being met corresponds to a volume $V_{i, j}$ in the space defined by the arrival times $t_{i}, i \in\{1, \ldots, N-1\}$. This space is equivalent to $\mathbb{R}_{++}^{N-1}$.

Exactly $k$ aircraft safely transiting the sector corresponds to a complicated region $V_{k}$ in $\mathbb{R}_{++}^{N-1}$. Specifically,

$$
V_{k}=\left(\cap_{j=1}^{m} \cap_{i=1}^{k-1} V_{i, j}\right) \cap\left(\cup_{j=1}^{m} \overline{V_{k, j}}\right) .
$$

Set complement is denoted by the overline $\left(\bar{S}=\mathbb{R}_{++}^{N-1} \backslash S\right)$. The distribution of arrival times is denoted $f(t)$, where $\mathbf{t}=\left(t_{1}, \ldots, t_{N-1}\right)$. The probability of exactly $k$ aircraft safely transiting the sector is $P_{k}=\int_{V_{k}} f(\mathbf{t}) d \mathbf{t}$.

\section{A. Single-link sector worst-case expressions}

Expressions for $P_{k}$ are obtained for single-link sectors for each of the three presented control strategies. The $i^{t h}$ interarrival time is the spacing between aircraft $i-1$ and $i$ and is denoted $\tau_{i}$. Thus, $\tau_{i}=t_{i}-t_{i-1}$ and $t_{i}=\sum_{j=1}^{i} \tau_{j}$. For $\boldsymbol{\tau}=\left(\tau_{1}, \ldots, \tau_{N-1}\right)$, the transformation from $\mathbf{t}$ to $\boldsymbol{\tau}$ is an isomorphism on $\mathbb{R}_{++}^{N-1}$. The region $V_{k}$ can therefore be described in terms of $\tau$. Also, the arrival process is often specified by the distribution of interarrival times, $f(\boldsymbol{\tau})$. Therefore, it is often convenient to work in terms of $\tau$. The probability that exactly $k$ aircraft transit the sector safely is then

$$
P_{k}=\int_{V_{k}} f(\boldsymbol{\tau}) d \boldsymbol{\tau} .
$$

Under the assumption the interarrival times $\tau_{i}, i \in$ $\{1, \ldots, N-1\}$ are independent, $f(\boldsymbol{\tau})$ factors as $\prod_{i=1}^{N-1} f_{i}\left(\tau_{i}\right)$, where the $f_{i}$ are marginal probability distributions.

In this paper, it is assumed that interarrival times are independent, which may be reasonable if upstream traffic is relatively sparse or controlled only to meet separation requirements, as is the case today. However, under more complex controls, upstream traffic may exhibit correlation between interarrival times. Under such conditions, a general expression for $f(\boldsymbol{\tau})$ must be used, but equations (10) and (11) still hold.

In each of the control strategies, aircraft $i$ can transit the sector safely if two conditions are met: (1) minimum velocity constraints and (2) sufficient separation between aircraft $i-1$ and $i$ when aircraft $i$ enters. These conditions lead to two constraints on the feasibility of the $i^{t h}$ aircraft: $C_{i, 1}$ and $C_{i, 2}$.

1) Fixed velocity trajectories: The first control strategy assigns a fixed velocity within $10 \%$ of nominal for the duration of the alignment phase. Aircraft $i$ meets minimum velocity requirements if $t_{i} \geq \frac{-2 D}{3}+\beta i$. The requirement that aircraft $i-1$ not interfere with the entry of aircraft $i$ can be written as $5 t_{i-1}+D \tau_{i} \geq 30 D+5 \beta(i-1)$. Transforming into interarrival coordinates yields the constraints

and

$$
C_{i, 1}: \sum_{j=1}^{i} \tau_{j} \geq \frac{-2 D}{3}+\beta i .
$$

$$
C_{i, 2}: 5 \sum_{j=1}^{i-1} \tau_{j}+D \tau_{i} \geq 30 D+5 \beta(i-1) .
$$

$V_{k}$ can thus be defined according to Equation (10). Integrating according to Equation (11) yields the probability that exactly $k$ aircraft can safely transit the sector. Solving for all $k$ yields the desired distribution.

2) Piecewise combination of nominal and slow trajectories: The constraints $C_{i, j}$ are developed for the second control policy using the same method as above:

$$
C_{i, 1}: \sum_{j=1}^{i} \tau_{j} \geq \frac{-2 D}{3}+\beta i
$$

and

$$
C_{i, 2}: \sum_{j=1}^{i} \tau_{j} \geq \frac{-2 D}{3}+\beta i+\left(\frac{100}{3}-\beta\right)
$$

In this case, only one constraint is active for given $\beta$. Again, Equations (10) and (11) are used to calculate the probability of exactly $k$ aircraft safely transiting the sector.

3) Vector-for-spacing trajectories: The third control policy yields a further set of constraints $C_{i, j}$ :

$$
C_{i, 1}: \sum_{j=1}^{i} \tau_{j} \geq \frac{-2(10 \sqrt{2}-9) D}{3}+\beta i .
$$

and

$$
C_{i, 2}: \sum_{j=1}^{i} \tau_{j} \geq \frac{-2(10 \sqrt{2}-9) D}{3}+\beta i+\left(\frac{100 \sqrt{2}}{3}-\beta\right) .
$$

The integration in Equation (11) is difficult for any of the above cases, because constraints are not independent, yielding a complex region $V$. Numerical integration is required to directly compute the desired distribution $P_{k}$; this only yields an approximation to the distribution. However, Monte Carlo simulation can also be used to estimate $P_{k}$. Indeed, the simulation results that follow are used to generate estimates of $P_{k}$.

\section{B. Simulation results}

In Section III, a single simulation is used to show failure of the slot-based sector model. For general arrival processes, Monte Carlo simulations are used to estimate the distribution of worst-case capacity. For each parameter set, 5000 simulations are run to generate a histogram of worst-case results. 
That is, each simulation gives a number of aircraft handled until failure; these numbers are then tabulated to estimate the distribution of worst-case capacity.

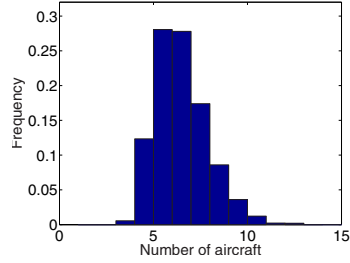

(a)

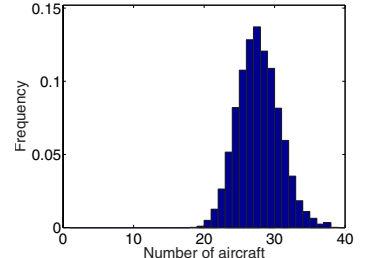

Fig. 4. Worst-case results via Monte Carlo simulation for single-link sector, fixed-spacing control policy, with $L=100 \mathrm{~nm}, D=20 \mathrm{~nm}, \beta=60$ seconds, and arrivals according to a distribution uniform on (a) $[55,65]$, (b) $[59,61]$ seconds.

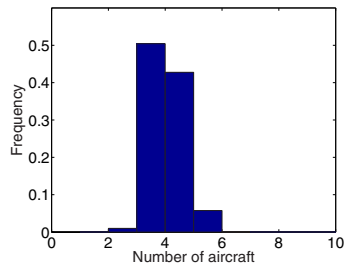

Fig. 5. Identical to 4 (a) with outflow spacing $\beta=62$ seconds.

The first set of results are for a single-link sector with length $L=100 \mathrm{~nm}$, alignment phase length $D=20 \mathrm{~nm}$, outflow spacing $\beta=60$ seconds, and a uniform distribution between 55 and 65 seconds for the interarrival times. Because the average inflow spacing is 60 seconds, this scenario corresponds to smoothing the flow of traffic, not adding overall delay. The histogram of worst-case capacities is shown in Figure 4(a) for the first control policy, fixed-velocity. The second control policy performs very similarly, while the vector-for-spacing policy performs significantly better.

Results from a second simulation are shown in Figure 4(b). This simulation is identical to the first except that interarrival times are uniformly distributed between 59 and 61 seconds. The narrower arrival distribution makes it easier to handle incoming aircraft, leading to a right-shifted distribution of worst-case capacities.

In the third simulation (results in Figure 5), the original interarrival distribution is used (uniform on $[55,65]$ ), but the desired outflow spacing is set to 62 seconds. In this scenario, the aircraft flow is both smoothed and delayed. As expected, this scenario is more difficult to control and the distribution of worst-case capacities is shifted left.

Different sector geometries and control policies can be analyzed in terms of worst-case capacities, even under uncertainty in the arrival process. Simulation results presented above show that different conditions lead to drastically different expected worst-case scenarios. Half of the trials in Figure 5 result in only handling 3 aircraft successfully while more than half the trials in Figure 4(b) result in handling over 25 aircraft successfully. Even for a specific arrival process, the outcome may be very varied: in Figure 4(a), between 4 and 11 aircraft may be handled safely. However, narrower interarrival distributions, which correspond to reduced uncertainty in aircraft arrivals, improve the efficacy of the slotbased sector model. That is, control becomes easier with added knowledge. This idea is explored further through a discussion of controller workload in the next section.

\section{AIR TRAFFIC CONTROLLER WORKLOAD RESULTS}

In previous sections, analytic and simulation results are presented to show the efficacy of the slot-based sector model under a variety of inflow conditions. However, by forcing aircraft to align to and then maintain slots, superior control strategies may be ignored. Intuitively, a restrictive structure is beneficial by reducing air traffic controller workload.In this section, this intuition is explored by first defining a metric for controller workload, then using the metric to compare the performance of the slot-based model under different inflows and alignment control policies.

\section{A. Metric to measure controller workload}

Many factors contribute to controller workload; a variety of quantitative models have been developed to measure workload [10], [11], [12], [13], [14]. A simple, new metric is proposed: a linear combination of only four factors suggested in existing models. Because of the dynamic nature of the system, each term is defined over a two-minute horizon.

First, controllers must monitor and possibly communicate with each flight in the sector; therefore, the sector count is a relevant factor in measuring controller workload. Consider all aircraft in the system $\left(i \in\left\{1, \ldots, N^{*}\right\}\right)$, and let the current time (in seconds) be $t_{0}$. Define the probability that aircraft $i$ is in the sector in the next two minutes as $c_{i}=\sup _{t \in\left[t_{0}, t_{0}+120\right]} \operatorname{Prob}($ aircraft $i$ in sector at time $t)$. Then sector count $C$ is defined as $C=\sum_{i=1}^{N^{*}} c_{i}$.

Speed, heading, and altitude changes require additional controller attention. This factor, denoted $\Delta$, is calculated by counting the expected number of trajectory changes in the next two minutes for each aircraft $i \in\left\{1, \ldots, N^{*}\right\}$, scaling each by $c_{i}$, and summing.

The third term, $\Gamma$, represents a count of the number of pairs of aircraft within $15 \mathrm{~nm}$ of each other in the next two minutes; aircraft near each other require controller monitoring. The minimum distance between aircraft $i$ and $j$ is defined as $d_{i j}=\inf \left\{\left|x_{i}(t)-x_{j}(t)\right|, t \in\left[t_{0}, t_{0}+120\right]\right\}$. The variable $\Gamma$ is defined as $\sum_{i=1}^{k} \sum_{j=1, j \neq i}^{k} c_{i} c_{j} I\left(d_{i j}<15\right)$, where $I(x)$ is the indicator function: 0 if $x$ is false and 1 if $x$ is true.

The last term considered measures the uncertainty of aircraft positions. Over a two minute horizon, aircraft entering the sector or aircraft placed on conflict avoidance trajectories may have uncertain positions. Such uncertainty requires attention from controllers. The variance of the position of aircraft $i$ at time $t_{0}+120$ is denoted $\sigma_{i}^{2}$. The total uncertainty term is denoted $S$ and defined as $S=\sum_{i=1}^{N^{*}} c_{i} \sigma_{i}^{2}$.

The controller workload, then, is

$$
W=\eta_{1} C+\eta_{2} \Delta+\eta_{3} \Gamma+\eta_{4} S .
$$

The constants $\eta_{i}, i \in 1, \ldots, 4$ are calibrated against data but are free parameters for the purposes of this discussion.

\section{B. Derivation of controller workload results}

Workload for a set of arrival processes is determined using the metric above. Consider a single-link sector with link length $L=100 \mathrm{~nm}$, alignment phase length $D=20 \mathrm{~nm}$, and exit spacing $\beta=60$ seconds; interarrival times have a uniform distribution over $[60-s, 60+s]$. The configuration is as in Figure 6, where aircraft 10 has just entered the 
sector. In two minutes, aircraft 11 will enter the sector, and aircraft 12 has a $50 \%$ chance of entering the sector. Therefore, $c_{i}=1, i \in\{1, \ldots, 11\}$ and $c_{12}=0.5$, and the sector count is $C=11.5$.

Aircraft in maintenance requires zero trajectory changes, while aircraft in the alignment phase or entering aircraft all require one velocity change. Scaling by $c_{i}$, we find $\Delta=4.5$.

10 pairs of aircraft (aircraft $i$ and $i+1$ for $i \in\{1, \ldots, .10\}$ are in the sector and within $15 \mathrm{~nm}$. Aircraft 11 and 12 are closer than $15 \mathrm{~nm}$, but $c_{12}=0.5$; therefore, $\Gamma=10.5$.

Finally, aircraft positions are known exactly except for aircraft 11 and 12. Aircraft 11 enters at a time $t_{11} \in[60-$ $s, 60+s]$, and has velocity $v_{11}=600 * 6 D /\left(6 D+66-t_{11}\right)$ $\mathrm{nm} / \mathrm{hr}$. The position 120 seconds after the current time is then $x_{11}\left(t_{\text {curr }}+120\right)=v_{11} *\left(120-t_{11}+t_{\text {curr }}\right) / 3600$, a function of $t_{11}$. Position variance is found to be $\sigma_{11}^{2}=2.31 * 10^{-3} \mathrm{~s}^{2}$, within $1 \%$ accuracy. Similarly, to within $1 \%$ accuracy, $\sigma_{12}^{2}=$ $1.52 * 10^{-4} s^{2}$. Therefore, $S \approx 2.46 * 10^{-3} s^{2}$.

$S$ is an increasing function of $s$. The other terms in Equation (18) are constants; therefore controller workload $W$ increases with $s$. The quantity $s$ represents the uncertainty in interarrival times; as expected, increasing arrival variability increases controller workload.

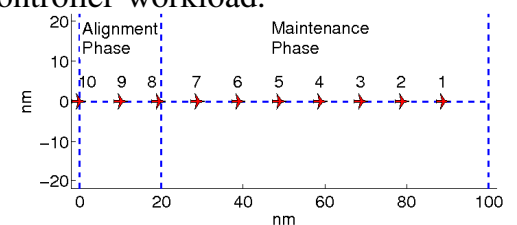

Fig. 6. Configuration of aircraft in single-link sector scenario, with sector parameters $L=100 \mathrm{~nm}, D=20 \mathrm{~nm}, \beta=60$, and interarrival times uniform on $[60-s, 60+s]$.

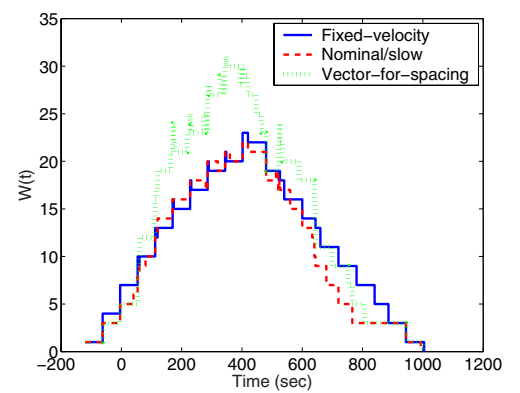

Fig. 7. Workload $\mathrm{W}(\mathrm{t})$ plotted over time for three proposed control policies. Simulation of single-link sector with $L=100 \mathrm{~nm}, D=20 \mathrm{~nm}, \alpha=58$, and $\beta=60$.

\section{Controller workload simulation results}

Next, a specific scenario is simulated to observer the evolution of controller workload under the different alignment control policies. Again consider a single-link sector with $L=100 \mathrm{~nm}$, alignment phase length $D=20 \mathrm{~nm}$, and exit spacing $\beta=60$ seconds, but the entry spacing is set to a constant 58 seconds. The first of 10 aircraft enters at time 0 , and the simulation continues until all aircraft have traversed the sector. Setting the constants $\eta_{i}$ to unity, controller workload is obtained as a function of time, $W(t)$, as shown in Figure 6. The vector-for-spacing policy results in much higher peak controller workload than the other two policies. More complicated trajectories increase the $\Delta$ term representing aircraft trajectory changes, while other terms are relatively unchanged. As a whole, controller workload increases under complicated alignment policies. Further studies are warranted to better understand the relationship between the arrival process and controller workload.

\section{CONCLUSION}

A novel slot-based sector model in used for enroute airspace flow control. The model splits a sector into an alignment phase (in which possibly complex trajectories are used to safely align aircraft to slots) and a maintenance phase (in which aircraft travel on slots at nominal speed through the sector with no possibility of safety violation). Increased uncertainty in arrivals is shown to reduce worst-case capacities and increase controller workload. More complex alignment policies such as vector-for-spacing are found to increase capacity at the expense of increased controller workload.A more general framework of analysis may be needed to extend results to arbitrary sector geometries. Also of interest is applying the slot-based sector model over multiple sectors to analyze larger regions of the National Airspace System.

\section{REFERENCES}

[1] A. M. Bayen, P. Grieder, G. Meyer, and C. J. Tomlin, "Lagrangian delay predictive model for sector-based air traffic flow," in Proc. AIAA Guidance, Navigation, and Control Conference, Providence, RI, August 2004.

[2] P. K. Menon, G. D. Sweriduk, T. Lam, V. H. L. Cheng, and K. BILIMORIA, "Air traffic flow modeling, analysis and control," in Proc. AIAA Conference on Guidance, Navigation and Control, Austin, TX, Aug. 2003, paper 2003-5712.

[3] J. KUCHAR and L. YANG, "A review of conflict detection and resolution modeling methods," IEEE Transactions on Intelligent Transportation Systems, vol. 1, no. 4, pp. 179-189, 2000.

[4] D. Dugail, Z.-H. MaO, and E. Feron, "Stability of intersecting aircraft flows under centralized and decentralized conflict avoidance rules," in Proc. AIAA Conference on Guidance, Navigation, and Control, Montreal, Canada, Aug. 2001.

[5] D. Dugail, E. Feron, and K. Bilimoria, "Conflict-free conformance to En-Route flow-rate constraints," in Proc. AIAA Conference on Guidance, Navigation and Control, Monterey, CA, Aug. 2002.

[6] L. Pallottino, A. Bicchi, and E. Feron, "Mixed Integer Programming for aircraft conflict resolution," in Proc. AIAA Guidance, Navigation, and Control Conference, Montreal, Canada, Aug. 2001.

[7] S. ChATTY, H. UNINSKI, and S. SiRe, "Supporting collaboration in ATC through flight contracts," in Proc. 2nd USA/Europe Air Traffic Management $R \& D$ seminar, Orlando, FL, Dec. 1998.

[8] M. Le Guillou, B. Cazard, H. De Jonge, R. Maddock, J.-P. NICOLAON, and R. MCGREGOR, "Pd/3 operational scenario document," Programme for Harmonized Air Traffic Management Research in EUROCONTROL, Tech. Rep. 97-70-04, Jan. 1997.

[9] V. AGUADO, "Opening address," presented at the Boeing ATMAirports Workshop, Gijon, Spain, Jan. 2004.

[10] G. ChatTerJI and B. SRIDHAR, "Measures for air traffic controller workload prediction," in Proc. 1st AIAA Aircraft, Technology, Integration, and Operations Forum, Los Angeles, CA, Oct. 2001.

[11] B. SRIDHAR, K. Sheth, and S. GRABbE, "Airspace complexity and its application in air traffic management," in Proc. 2nd USA/Europe Air Traffic Management R\&D seminar, Orlando, FL, Dec. 1998.

[12] D. Delahaye and S. Puechmorel, "Air traffic complexity: towards intrinsic metrics," in Proc. 3rd USA/Europe Air Traffic Management $R \& D$ seminar, Naples, Italy, June 2000.

[13] A. Masalonis, M. Callaham, and C. Wanke, "Dynamic density and complexity metrics for realtime traffic flow management," MITRE, Tech. Rep., Sep. 2003.

[14] P. KopardekAR and S. Magyarits, "Dynamic density: Measuring and predicting sector complexity," presented at the 21st Digital Avionics Systems Conference, Irvine, CA, 2002. 